\title{
Multivariate Regression Depth
}

\author{
Marshall Bern \\ Xerox Palo Alto Research Center \\ 3333 Coyote Hill Rd. \\ Palo Alto, CA 94304, USA \\ bern@parc.xerox.com
}

\author{
David Eppstein \\ Dept. of Information and Computer Science \\ University of California, Irvine \\ Irvine, CA 92697, USA \\ eppstein@ics.uci.edu
}

\begin{abstract}
The regression depth of a hyperplane with respect to a set of $n$ points in $\mathbb{R}^{d}$ is the minimum number of points the hyperplane must pass through in a rotation to vertical. We generalize hyperplane regression depth to $k$-flats for any $k$ between 0 and $d-1$. The $k=0$ case gives the classical notion of center points. We prove that for any $k$ and $d$, deep $k$-flats exist, that is, for any set of $n$ points there always exists a $k$-flat with depth at least a constant fraction of $n$. As a consequence, we derive a linear-time $(1+\epsilon)$-approximation algorithm for the deepest flat.
\end{abstract}

\section{INTRODUCTION}

Linear regression asks for an affine subspace (a flat) that fits a set of data points. The most familiar case assumes $d-1$ independent or $e x$ planatory variables and one dependent or response variable, and fits a hyperplane to explain the dependent variable as a linear function of the independent variables. Quite often, however, there may be more than one dependent variable, and the multivariate regression problem requires fitting a lower-dimensional flat to the data points, perhaps even a succession of flats of increasing dimensions. Multivariate least-squares regression is easily solved by separately fitting each dependent variable, but this is not correct for other common forms of regression such as least sum of errors [7] or least median of squares [13].

Rousseeuw and Hubert [15] introduced regression depth as a robust criterion for linear regression. The regression depth of a hyperplane $H$ fitting a set of $n$ points is the minimum number of points whose removal makes $H$ into a nonfit. A nonfit is a hyperplane that can be rotated to vertical (that is, parallel to the dependent variable's axis) without passing through any points. The intuition behind this definition is that a vertical hyperplane posits no relationship between the dependent and independent variables, and hence many points should have to be invalidated in order to make a good regression hyperplane combinatorially equivalent to a vertical hyperplane. Since this definition does not make use of the size of the residuals, but only their signs, it is robust in the face of skewed or heteroskedastic (data-dependent) error models. Regression depth also has a number of other nice properties including invariance under affine transformations, and a connection to the classical notions of center points and data depth.

This paper generalizes regression depth to the case of more than one dependent variable, that is, to fitting a $k$-flat to points in $\mathbb{R}^{d}$. This generalization is not obvious: for example, consider fitting a line to points in $\mathbb{R}^{3}$. Generic lines can be rotated wherever one likes without passing through data points, so how are we to distinguish one line from another?

We start by reviewing previous work (Section 2) and stating our basic definitions (Section 3 ). We then provide a lemma that may be of independent interest, on finding a family of large subsets of any point set such that the family has no hyperplane transversal (Section 4). We prove the existence of deep $k$-flats for any $k$ (Section 5), and give tight bounds on depths of lines in $\mathbb{R}^{3}$ (Section 6). We conclude by discussing related generalizations of Tverberg's theorem (Section 7), describing a possible duality between regression depth for $k$-flats and $(d-k-1)$-flats (Section 8$)$, and outlining the algorithmic implications of our existence proof (Section 9). Along with the results proven in each section, we list open problems for further research.

\section{PREVIOUS WORK}

Regression depth was introduced by Rousseeuw and Hubert [15] as a combinatorial measure of the quality of fit of a regression hyperplane. An older notion, variously called data depth, location depth, halfspace depth, or Tukey depth, similarly measures the quality of fit of a single-point estimator. It has long been known that there exists a point of location depth at least $\lceil n /(d+1)\rceil$ (a center point). Rousseeuw and Hubert provided a construction called the catline [4] for computing a regression line for a planar point set with depth at least $\lceil n / 3\rceil$ in linear time, and conjectured [14] that in higher dimensions as well there should always exist a regression hyperplane of depth $\lceil n /(d+1)\rceil$. Steiger and Wenger [17] proved that a deep regression hyperplane always exists, but with a much smaller fraction than $\lceil 1 /(d+1)\rceil$. Amenta, Bern, Eppstein, and Teng [2] solved the conjecture using an argument based on Brouwer's fixed-point theorem and a close connection between regression depth and center points; independently, Mizera [9] also proved Rousseeuw and Hubert's conjecture.

On the algorithmic front, Rousseeuw and Struyf [16] found methods for testing the regression depth of hyperplanes. Their time bounds are exponential in the dimension, unsurprising since the problem is 
NP-complete for unbounded dimension [2]. Van Kreveld, Mitchell, Rousseeuw, Sharir, Snoeyink, and Speckmann gave an $O\left(n \log ^{2} n\right)$ algorithm for computing a deepest line in $\mathbb{R}^{2}$ [5], and Langerman and Steiger [6] improved this to an optimal $O(n \log n)$ time bound.

\section{DEFINITIONS}

Although regression is naturally an affine rather than projective concept, our constructions and definitions live most gracefully in projective space. We view $d$-dimensional real projective space as a renaming of objects in $(d+1)$-dimensional affine space. (Affine space is the familiar Euclidean space, only we have not specified a distance metric.) A $k$-flat, for $1 \leq k \leq d$, through the origin of $(d+1)$ dimensional affine space is a projective $(k-1)$-flat. In particular a line through the origin is a projective point and a plane through the origin is a projective line. A projective line segment is the portion of a projective line between two projective points, that is, a pair of opposite planar wedges with vertex at the origin.

We can embed affine $d$-space into projective space as a hyperplane that misses the origin. There is a unique line through any point of this hyperplane and the origin, and hence each point of affine space corresponds to a unique projective point. There is, however, one projective hyperplane, and many projective $k$-flats for $k<d-1$, without corresponding affine flats; these are the projective $k$-flats parallel to the affine space. We say that these flats are at infinity.

Each projective point $p$ corresponds to a dual projective hyperplane $D(p)$, namely the hyperplane orthogonal to $p$ at the origin in $(d+1)$ dimensional affine space. Similarly a projective $k$-flat dualizes to its orthogonal $(d-k)$-flat. Notice that in projective space, unlike in affine space, there are no exceptional cases: each $k$-flat is the dual of a $(d-k)$-flat.

Now let $X$ be a set of points in $d$-dimensional projective space. From now on we shall just say "point", "line", etc. rather than "projective point", "projective line", when there is no risk of confusion. We now propose a key definition: a distance between flats with respect to the points in $X$. The definition is more intuitive in the dual formulation than in the primal, but we give both below for completeness. Let $D(F)$ denote the flat that is dual to flat $F$ and let $D(X)$ denote the set of hyperplanes that are dual to points of $X$. A double wedge is the (closed) region between two projective hyperplanes.

DEFINITION 1. The crossing distance between two flats $F$ and $G$ with respect to $X$ is the minimum number of hyperplanes of $D(X)$ intersected by a closed projective line segment with one endpoint on $D(F)$ and the other on $D(G)$. In the primal formulation, the crossing distance between $F$ and $G$ is the minimum number of points of $X$ in a double wedge that contains $F$ in one bounding hyperplane and $G$ in the other.

We now turn our attention to linear regression and for ease of understanding, we return temporarily to $d$-dimensional affine space. Assume that we designate $k$ dimensions as independent variables and $d-k$ as dependent variables. Let $I$ denote the linear subspace spanned by the independent dimensions. We call a $k$-flat vertical if its projection onto $I$ is not full-dimensional, that is, if its projection is not all of $I$. For example, let $k=1$ and $d=3$ and think of the $x$-axis as representing the independent variable; then any line contained in a vertical plane (that is, parallel to the $y z$-plane) is vertical.
In projective space, a $k$-flat is vertical if and only if it contains a point in a particular $(d-k-1)$-flat at infinity, which we call the $(d-k-1)$-flat at vertical infinity and denote by $V_{d-k-1}$.

DEFINITION 2. The regression depth of a $k$-flat $F$ is its crossing distance from $V_{d-k-1}$. Equivalently, the regression depth of $F$ is the minimum number of points whose removal makes $F$ into a nonfit, where a nonfit is a $k$-flat with crossing distance zero from $V_{d-k-1}$.

Any $k$-flat at infinity meets $V_{d-k-1}$ and therefore has depth zero. Therefore, any method for selecting a $k$-flat of nonzero regression depth will automatically choose a $k$-flat coming from the original affine space, rather than one that exists only in the projective space used for our definitions.

Note that, unlike the case for ordinary least squares, there does not seem to be any way of solving $k$-flat regression separately for each dependent variable. Even for the problem of finding a line in $\mathbb{R}^{3}$, combining the solution to two planar regression lines may result in a nonfit.

\section{NONTRANSVERSAL FAMILIES}

In order to prove that deep $k$-flats exist, we need some combinatorial lemmas on large subsets of points without a hyperplane transversal.

DEFINITION 3. Let $S$ be a set of points in $\mathbb{R}^{d}$. Then we say that a hyperplane $H$ cuts $S$ if each of the two open halfspaces bounded by $H$ contains at least one point of $S$. We say that a family of sets is transversal if there is a hyperplane that cuts all sets in the family.

LEMma 1 (YAO, YAO, AND MATOUŠEK $[8,20]$ ). Let d be a constant, and assume we are given a set of $n$ points in $\mathbb{R}^{d}$ and a parameter $p$. Then we can partition the points into $p$ subsets, with at most $2 n / p$ points in each subset, such that any hyperplane cuts $o(p)$ of the subsets.

Lemma 2 (ALON AND KALAI [1]). Let $p \geq q>d$ be constants. Then there is a constant $C(p, q, d)$ with the following property: If $\mathcal{F}$ is any family of point sets in $\mathbb{R}^{d}$, such that any p-tuple of sets in $\mathcal{F}$ contains a transversal subfamily of $q$ sets, then $\mathcal{F}$ can be partitioned into $C(p, q, d)$ transversal subfamilies.

THEOREM 1. Let d be a constant. Then there is a constant $P(d)$ with the following property: For any set $S$ of n points in $\mathbb{R}^{d}$, we can find a nontransversal family of $d+1$ subsets of $S$, such that each subset in the family contains at least $\lceil n / P(d)\rceil$ points of $S$.

Proof. Choose $p$ to be a multiple of three, sufficiently large that the $o(p)$ bound of Lemma 1 is strictly smaller than $p /(3 C(d+1, d+$ $1, d)$ ), and let $P(d)=2 p$. By Lemma 1 , partition $S$ into $p$ subsets of at most $2 n / p$ points, such that any hyperplane cuts few subsets.

Let $\mathcal{F}$ be the family consisting of the largest $p / 3$ subsets in the partition. If the smallest member of $\mathcal{F}$ contains $m$ points, then the total size of all the members of the partition would have to be at most $(p / 3) \cdot 2 n / p+(2 p / 3) \cdot m=2 n / 3+2 p m / 3$, but this total size is just $n$, so $m \geq n /(2 p)$ and each member of $\mathcal{F}$ contains at least $n / P(d)$ points. 

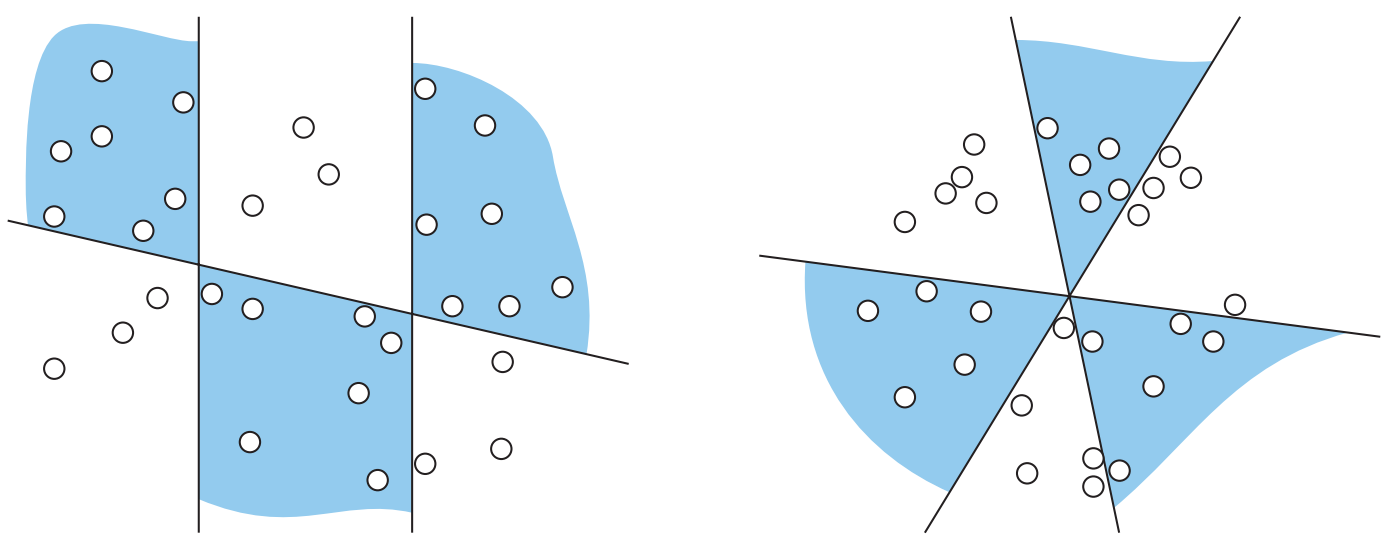

Figure 1: Construction of three nontransversal sets of $n / 6$ points in $\mathbb{R}^{2}$ : (a) catline, formed by partitioning the point set vertically into equal thirds, and making a ham sandwich cut of the leftmost and rightmost $2 n / 3$ points; (b) subdivision by three coincident lines into equal sixths.

If each $(d+1)$-tuple of sets in $\mathcal{F}$ were transversal, we could apply Lemma 2 and partition $\mathcal{F}$ into $C(p, q, d)$ transversal subfamilies, one of which would have to contain at least $|\mathcal{F}| / C(p, q, d)=$ $p /(3 C(d+1, d+1, d))$ subsets. But this violates the $o(p)$ bound of Lemma 1 , so $\mathcal{F}$ must contain a nontransversal $(d+1)$-tuple. This tuple fulfills the conditions of the statement of the lemma.

Clearly, $P(1)=2$ since the median partitions any set of points on a line into two nontransversal subsets. Figure 1 depicts two different constructions showing that $P(2) \leq 6$. Although the bound of six is tight for these two constructions (as can be seen by the example of points equally spaced on a circle) we do not know whether there might be a different construction that achieves a better bound; the best lower bound we have found is the following:

THEOREM 2. $P(2) \geq \frac{\pi}{2 \sin ^{-1} \frac{1}{3}} \approx 4.622$.

PROOF. We form a distribution on the plane by centrally projecting the uniform distribution on a sphere. We show that any nontransversal triple for this distribution must have a set with measure at most $1 / 4.622$ times the total measure. The same bound then holds in the limit for discrete point sets formed by taking $\epsilon$-approximations of this distribution.

Let $S_{i}, i \in\{1,2,3\}$ denote the three nontransversal subsets of the plane maximizing the minimum measure of any $S_{i}$. Without loss of generality, each $S_{i}$ is convex. Consider the three lines tangent to two of the $S_{i}$, and separating them from the third set (such a line must exist since the sets are nontransversal). These lines form an arrangement with seven (possibly degenerate) faces: a triangle adjacent on its edges to three three-sided infinite cells, and on its vertices to three two-sided infinite cells. The sets $S_{i}$ coincide with the threesided infinite cells: any set properly contained in such a cell could be extended to the whole cell without violating the nontransversality condition, and if they instead coincided with the two-sided cells we could shrink the arrangement's central triangle while increasing the sizes of all three $S_{i}$. The two arrangements in Figure 1 can both be viewed as such three-line arrangements, degenerate in different ways.
Each line in the plane lifts by central projection to a great circle on the sphere. Consider the great circles formed by lifting four lines: the three lines considered above and the line at infinity. Any arrangement of four circles on the sphere cuts the sphere in the pattern of a (possibly degenerate) cuboctahedron (Figure 2(a)). The three-sided infinite cells in the plane lift to quadrilateral faces of this cuboctahedron. Note that the area of a spherical quadrilateral is the sum of its internal angles, minus $2 \pi$.

Form the dual of the arrangement by treating each great circle as an "equator" and placing a pair of points at the corresponding two poles. The geodesics between these points form a cuboid pattern (Figure 2(b)) such that the length of each geodesic is an angle complementary to one of the cuboctahedron quadrilaterals' internal angles. Thus, the cuboid minimizing the maximum quadrilateral perimeter corresponds to the cuboctahedron maximizing the minimum quadrilateral area. But any spherical cuboid has at least one face covering at least one-sixth of the sphere, and the minimum perimeter for such a quadrilateral is achieved when the quadrilateral is a square. Therefore, the regular cube minimizes the maximum perimeter and the regular cuboctahedron maximizes the minimum area. The ratio of a regular cuboctahedron's square face area to the area of a full hemisphere is the value given, $\frac{\pi}{2 \sin ^{-1} 1 / 3} \approx 4.622$.

We also do not know tight bounds on $P(d)$ for $d \geq 3$. The proof of Theorem 1 (using the best known bounds in Lemma 1 [8]) leads to upper bounds of the form $O\left(C(d+1, d+1, d)^{-d}\right)$. We may be able to improve this somewhat, to $O\left(C(d+1, d, d-1)^{1-d}\right)$, by a more complicated construction: Project the points arbitrarily onto a $(d-$ 1)-dimensional subspace, find a partition in the subspace, and use Lemma 2 to find a family $F$ of $d+1$ subsets such that no subfamily of $d$ subsets has a transversal. As in the catline construction [4], group these subsets into $d$ pairs, and form a ham sandwich cut in $\mathbb{R}^{d}$ that partitions each subset in $F$ in the same proportion $a: b$, and such that the half-subsets of size $a$ are above or below the ham sandwich cut accordingly as the members of the family of $d+1$ subsets are on one or the other side of a Radon partition of those subsets in $\mathbb{R}^{d-1}$. Without loss of generality, $a>b$; then choose the $d+1$ subsets required by Lemma 1 to be the ones of size $a$.

Open Problem 1. Prove tighter bounds on $P(d)$ for $d \geq 2$. 

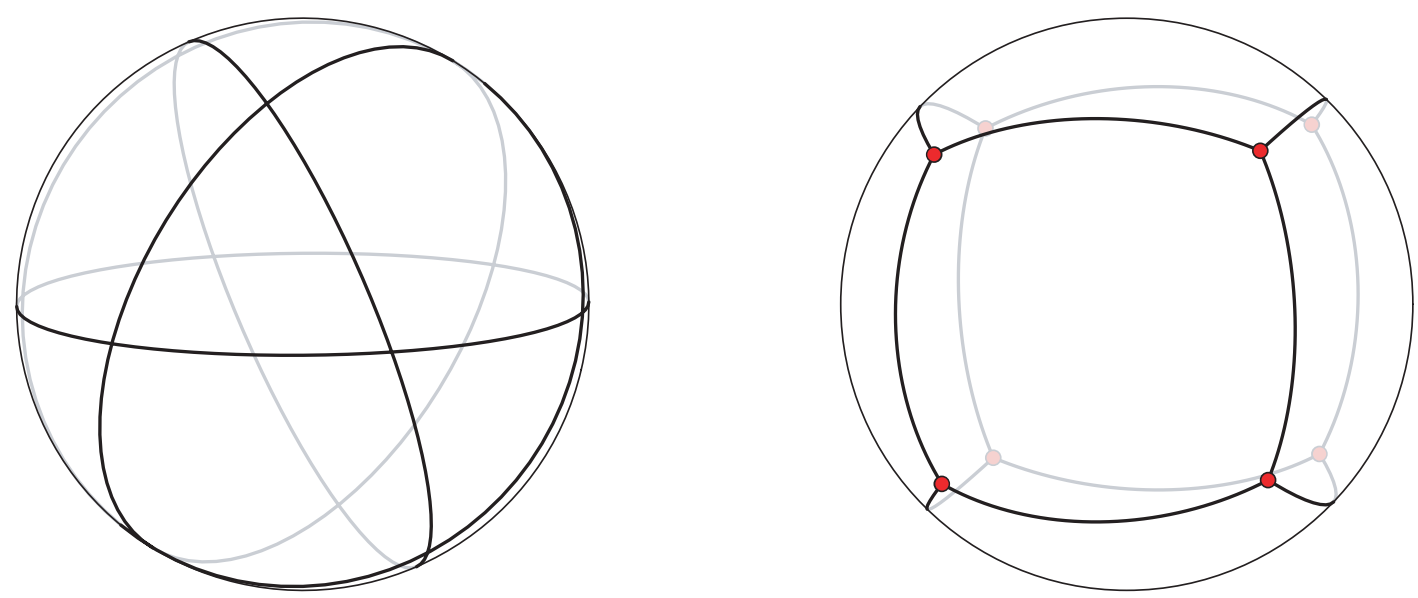

Figure 2: (a) Four great circles subdivide a sphere in the pattern of a cuboctahedron. (b) Four pairs of opposite points, connected by great circle arcs in the pattern of a cuboid.

\section{DEEP FLATS}

It is previously known that deep $k$-flats exist for $k=0$ [11] and $k=d-1[2,17]$. In this section we show that such flats exist for all other values of $k$.

We first need one more result, a common generalization of centerpoints and the ham sandwich theorem, known as the center transversal theorem:

LEMMA 3 (ŽIVALJEVIĆ, ET AL. [3,21]). Let $k+1$ point sets be given in $\mathbb{R}^{d}$, each containing at least m points, where $0 \leq k<d$. Then there exists a $k$-flat $F$ such that any closed halfspace containing $F$ contains at least $\lceil m /(d-k+1)\rceil$ points from each set.

The weaker bound $\lceil m /(d+1)\rceil$ can be proven simply by choosing a flat through the centerpoint of each subset.

THEOREM 3. Let $d$ and $0 \leq k<d$ be constants. Then there is a constant $R(d, k)$ such that for any set of $n$ points with $k$ independent and $d-k$ dependent degrees of freedom, there exists a $k$-flat of regression depth at least $\lceil n / R(d, k)\rceil$.

PROOF. Project the points vertically onto the subspace spanned by the $k$ independent directions, in such a way that the inverse image of each point in the projection is a $(d-k)$-flat containing $V_{d-k-1}$. By Theorem 1, we can find a family of $k+1$ subsets of the data points, each with $n / P(k)$ points, such that the $k$-dimensional projection of this family has no transversal. We then let $F$ be the $k$-flat determined by applying Lemma 3 to this family of subsets.

Then consider any double wedge bounded by a hyperplane containing $F$ and a hyperplane containing $V_{d-k-1}$. The vertical boundary of this double wedge projects to a hyperplane in $\mathbb{R}^{k}$, so it must miss one of the $k+1$ subsets in the family. Within this missed subset the double wedge appears to be simply a halfspace through $F$. By Lemma 3, the double wedge must therefore contain at least $n /((d-k+1) P(k))$ points. Thus if let $R(d, k)=(d-k+1) P(k)$ the theorem is satisfied.
For $k=0$ or $k=d-1$ we know that $R(d, k)=d+1$ [2]. However exact values are not known for intermediate values of $k$.

Open Problem 2. Prove tighter bounds on $R(d, k)$ for $1 \leq$ $k \leq d-2$.

The following conjecture would follow from the assumption that $R(d, k)$ is a linear function of $d$ for fixed $k$ (as the $O(d)$ bound of Theorem 3 makes plausible), since $R(k, k)=1$ and $R(k+1, k)=$ $k+2$. It also matches the known results $R(d, 0)=R(d, d-1)=$ $d+1$ and the bounds $R(d, 1) \leq 2 d-1$ and $R(3,1)=5$ of the following section. Finally, Ivan Mizera (personal communication) has pointed out that the conjectured bound is simply one plus the number of degrees of freedom of a $k$-flat in $\mathbb{R}^{d}$.

\section{Conjecture 1. $R(d, k)=(k+1)(d-k)+1$.}

\section{TIGHTER BOUNDS FOR LINES}

The method used in the proof of Theorem 3 shows that $R(d, 1) \leq$ $2 d$. This can be slightly improved using a technique of overlapping sets borrowed from the catline construction.

THEOREM $4 . R(d, 1) \leq 2 d-1$.

PROOF. The proof of Theorem 3 can be viewed as projecting the points onto a horizontal line, dividing the line into two rays at the median of the points, and applying the center transversal theorem to the two sets of $n / 2$ points contained in each ray. Instead, we project the points onto the horizontal line as before, but partition this line into three pieces: two rays containing $(d-1) n /(2 d-1)$ points each, and a line segment in the middle containing the remaining $n /(2 d-$ 1) points. We then apply the center transversal theorem to two sets $S_{1}$ and $S_{2}$ of $d n /(2 d-1)$ points each, formed by the points having a projection in the union of the middle segment and one of the two rays. This theorem finds a line such that no halfspace containing it has fewer than $n /(2 d-1)$ points in either of the sets $S_{i}$. We claim that this line has regression depth at least $n /(2 d-1)$. 

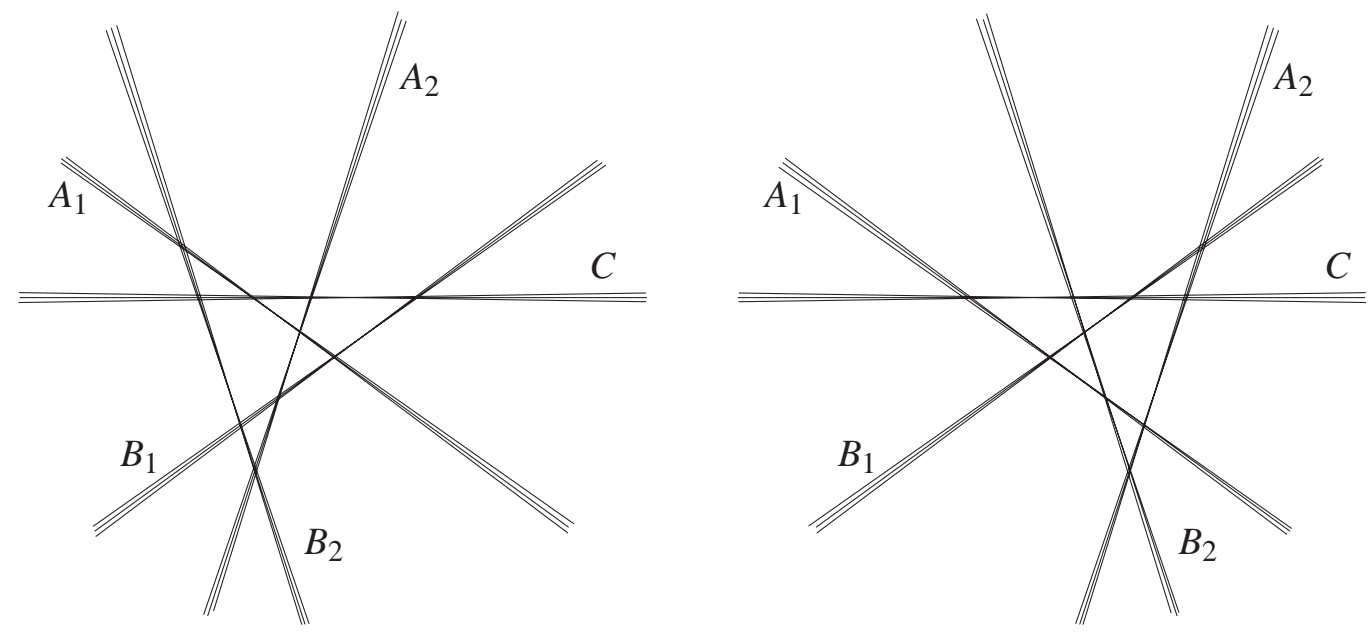

Figure 3: Three-dimensional plane arrangement showing $R(3,1) \geq 5$ : (a) cross-section for $x=1$; (b) cross-section for $x=-1$.

To prove this, consider any double wedge bounded by two hyperplanes, one containing the regression line and the other vertical. The vertical hyperplane intersects the horizontal projection line in a single point. If this intersection point is in one of the two rays, then the vertical hyperplane misses the set $S_{i}$ formed by the other ray and the middle segment. In this case, the double wedge contains the same subset of $S_{i}$ as a halfspace bounded by the double wedge's other bounding plane, and contains at least $n /(2 d-1)$ points of $S_{i}$

In the remaining case, the vertical boundary of the double wedge intersects the horizontal projection line in its middle segment. Within each set $S_{i}$, the double wedge differs from a halfspace (bounded by the same nonvertical plane) only within the subset of points projecting to this middle segment. But the halfspace corresponding to the double wedge in $S_{1}$ is complementary to the halfspace corresponding to the double wedge in $S_{2}$. Therefore, if we let $X_{i}$ denote the set of points in the halfspace but not in the double wedge for each set $S_{i}$, then $X_{1}$ and $X_{2}$ are disjoint subsets of the middle $n /(2 d-1)$ points. The number of points in the double wedge within $S_{i}$ must be at least $n /(2 d-1)-\left|X_{i}\right|$, so the total number of points in the double wedge is at least $2 n /(2 d-1)-\left|X_{1} \cup X_{2}\right| \leq 2 n /(2 d-1)-n /(2 d-1)=$ $n /(2 d-1)$.

Thus in all cases the double wedge contains at least $n /(2 d-1)$ points, showing that the line has depth at least $n /(2 d-1)$.

As evidence that this $2 d-1$ bound may be tight, we present a matching lower bound for $d=3$.

THEOREM 5. $R(3,1)=5$.

Proof. We have already proven that $R(3,1) \leq 5$, so we need only show that $R(3,1) \geq 5$. We work in the dual space, and construct an arrangement of $n$ planes in $\mathbb{R}^{3}$, for $n$ any multiple of 5 , such that any line has depth at most $n / 5$.

Our arrangement consists of five groups of nearly parallel closely spaced planes, which we label $A_{1}, A_{2}, B_{1}, B_{2}$, and $C$. Rather than describe the whole arrangement, we describe the line arrangements in the planar cross-sections at $x=1$ and $x=-1$. Recall that the depth of a line in the three-dimensional arrangement is the minimum number of planes crossed by any vertical ray starting on the line. Limiting attention to rays contained in the two cross-sections (and hence to the planar depth of the two points where the given line intersects these cross-sections) gives an upper bound on the depth of the line, and so a lower bound on $R(3,1)$.

In the first cross-section, we place the groups of lines as shown in Figure 3(a), with the region where $A_{1}$ and $A_{2}$ cross contained inside the triangle formed by the other three groups. Moreover, $A_{1}$ and $A_{2}$ do not cross side $B_{2}$ of the triangle, instead crossing group $B_{2}$ at points outside the triangle. The points where members of $A_{1}$ intersect each other are positioned on segment $C A_{1}-A_{1} A_{2}$. Similarly, the crossings within $A_{2}$ are situated on segment $A_{1} A_{2}-A_{2} B_{1}$. The crossings within $B_{1}, B_{2}$, and $C$ are situated along the corresponding sides of the triangle formed by these three groups.

In the cross-section formed as described above, points from most cells in the arrangement can reach infinity while crossing only one group, and so have depth at most $n / 5$. It is only within the segments $C A_{1}-A_{1} A_{2}$ and $A_{1} A_{2}-A_{2} B_{1}$ that a point can have higher depth. The arrangement is qualitatively similar for nearby cross-sections $x=1 \pm \epsilon$. Therefore, any deep line in $\mathbb{R}^{3}$ must be either nearly parallel to $A_{1}$ and not near any $B_{i}$, or nearly parallel to $A_{2}$ and not near $B_{2}$.

In the second cross-section (Figure 3(b)), the groups $A_{i}$ and $B_{i}$ reverse roles: the point where $B_{1}$ and $B_{2}$ cross is contained in the triangle determined by the other three groups, and the other details of the arrangement are situated in a corresponding manner. Therefore, any deep line would have to be either nearly parallel to $B_{1}$ and not near any $A_{i}$, or nearly parallel to $B_{2}$ and not near $A_{2}$.

There is no difficulty forming these two cross-sections from a single plane arrangement, since (as shown in Figures 3(a) and (b)) the slopes of the lines within each group can remain the same in each cross-section. But the requirements imposed on a deep line by these two cross-sections are contradictory, so no line can have depth more than $n / 5$ in this arrangement. 
We believe that a similar proof can be used to prove a more general $2 d-1$ lower bound on $R(d, 1)$ in any dimension, matching the upper bound in Theorem 4: form an arrangement with hyperplane groups $A_{i}, B_{i}$, and $C$, so that in one cross-section the $A_{i}$ meet in a vertex contained in a simplex formed by the other groups, and in the other cross-section the groups $A_{i}$ and $B_{i}$ exchange roles. However we have not worked out the details of where to place the intersections within groups, how to choose hyperplane angles such that the inner groups miss a face of the outer simplex in both cross-sections, or which cells of the resulting arrangements can have high depth.

\section{GENERALIZATIONS OF TVERBERG'S THEOREM}

A Tverberg partition of a set of point sites is a partition of the sites into subsets, the convex hulls of which all have a common intersection. The Tverberg depth of a point $t$ is the maximum cardinality of any Tverberg partition for which the common intersection contains $t$. Note that the Tverberg depth is a lower bound on the location depth. Tverberg's theorem $[18,19]$ is that there always exists a point with Tverberg depth $\lceil n /(d+1)\rceil$ (a Tverberg point); this result generalizes both the existence of center points (since any Tverberg point must be a center point) and Radon's theorem [12] that any $d+2$ points have a Tverberg partition into two subsets.

Another way of expressing Tverberg's theorem is that for any point set we can find both a partition into $\lceil n /(d+1)\rceil$ subsets, and a point $t$, such that $t$ has nonzero depth in each subset of the partition. Stated this way, there is a natural generalization to higher dimensional flats:

THEOREM 6. Let $d$ and $0 \leq k<d$ be constants. Then there is a constant $T(d, k)$ such that for any set of $n$ points with $k$ independent and $d-k$ dependent degrees of freedom, there exists $a k$-flat $F$ and a partition of the points into $\lceil n / T(d, k)\rceil$ subsets, such that $F$ has nonzero regression depth in each subset.

Proof. As in the proof of Theorem 3, we project the points onto the subspace spanned by the $k$ independent directions, in such a way that the inverse image of each point in the projection is a $(d-k)$ flat containing $V_{d-k-1}$. By Theorem 1, we can find a family of $k+$ 1 subsets $S_{i}$, each with $n / P(k)$ points, such that the $k$-dimensional projection of this family has no transversal. We then find a Tverberg point $t_{i}$ and a Tverberg partition of each set $S_{i}$ into subsets $T_{i, j}$, for $1 \leq j \leq\lceil n /(P(k)(d+1))\rceil$. We let $F$ be the $k$-flat spanning these $k+1$ Tverberg points. We form each set $T_{j}$ in our Tverberg partition as the union $\cup_{i} T_{i, j}$. Some points of $S$ may not belong to any set $T_{i, j}$, in which case they can be assigned arbitrarily to any set $T_{i}$.

Then consider any double wedge bounded by a hyperplane containing $F$ and a hyperplane containing $V_{d-k-1}$. The vertical boundary of this double wedge projects to a hyperplane in $\mathbb{R}^{k}$, so it must miss one of the $k+1$ subsets $S_{i}$. Within $S_{i}$ the double wedge appears to be simply a halfspace through $t_{i}$. It therefore contains at least one point of each set $T_{i, j}$ and a fortiori at least one point of each set $T_{j}$. Thus if let $R(d, k)=(d+1) P(k)$ the theorem is satisfied.

We know that $T(d, 0)=d+1$ by Tverberg's theorem, and the catline [4] shows that $T(2,1)=3$. However even in the case $k=d-1$ we do not know a tight bound; Rousseeuw and Hubert $[14,15]$ conjectured that $T(d, d-1)=d+1$ but the best bounds from our previous paper [2] are $T(d, d-1) \leq d(d+1)$ and $T(3,2) \leq 6$.
Open Problem 3. Prove tighter bounds on $T(d, k)$ for $1 \leq$ $k \leq d-1$.

\section{DUALITY}

There is a natural relation between finding a deep $k$-flat and finding a deep $(d-k-1)$-flat: in both cases one wants to find a $k$-flat and a $(d-k-1)$-flat that are far apart from each other, and the problems only differ in which of the two flats is fixed at vertical infinity, and which is to be found.

In our previous paper [2] we exploited this connection in the following way, to show that $R(d, d-1)=d+1$. A centerpoint (corresponding to $R(d, 0)$ ) is just a point far from a given "hyperplane at infinity"; in projective $d$-space, this hyperplane can be chosen arbitrarily, resulting in different centerpoint locations. We found an appropriate way to replace the input point set by a smooth measure, and modify the definition of a centerpoint, in such a way that we could show that the modified centerpoint location varied continuously as a function of the position of the hyperplane at infinity. We then used a variant of the Brouwer fixed point theorem to show that this function is surjective. A hyperplane in the inverse image of the point at vertical infinity is the desired deep regression plane.

Conjecture 1 implies that $R(d, k)=R(d, d-k-1)$, and one would naturally hope for a proof of this equality generalizing our previous proof that $R(d, d-1)=R(d, 0)$. Currently, the main obstacle is that we do not know how to modify the definition of a deep $k$-flat in such a way as to choose a unique flat which varies continuously as a function of the location of the $(d-k-1)$-flat at infinity. A similar lack of a continuous version of Tverberg's theorem blocked our attempts to prove that $T(d, d-1)=d+1$. We believe that if we can solve this technical difficulty, topological techniques should prove the essential equivalence of the $k$ and $d-k-1$ cases.

Open Problem 4. Does $R(d, k)=R(d, d-k-1)$ for $1 \leq$ $k \leq d-2$ ?

Open Problem 5. Does $T(d, k)=T(d, d-k-1)$ for $0 \leq$ $k \leq d-1$ ?

\section{ALGORITHMIC IMPLICATIONS}

We now show how to use our proof that deep flats exist as part of an algorithm for finding an approximate deepest flat. We begin with an inefficient exact algorithm.

THEOREM 7. Let $d$ and $k$ be constants. Then we can find the deepest $k$-flat for a collection of $n$ points in $\mathbb{R}^{d}$, in time $n^{O(1)}$.

Proof. Let $A$ be the arrangement of hyperplanes dual to the $n$ given points. The distance from points in $\mathbb{R}^{d}$ to $V_{d-k-1}$ is constant within each cell of $A$, and all such distances can be found in time $O\left(n^{d}\right)$ by applying a breadth first search procedure to the arrangement. The depth of a $k$-flat $F$ is just the minimum depth of any cell of $A$ pierced by $F$. Any two flats that pierce the same set of cells of $A$ have the same depth.

The space of $k$-flats forms a $(k+1)(d-k)$-dimensional algebraic set $F_{k}^{d}$, in which the flats touching any $(d-k-1)$-dimensional cell of $A$ 
form a subset of codimension one. The arrangement of these $O\left(n^{d}\right)$ subsets partitions $F_{k}^{d}$ into $O\left(n^{d(k+1)(d-k)+\epsilon}\right)$ cells, corresponding to collections of flats that all pierce the same set of cells. We can construct this arrangement, and walk from cell to cell maintaining a priority queue of the depths of the cells in $A$ pierced by the flats in the current cell, in time $O\left(n^{d(k+1)(d-k)+\epsilon}\right)$.

We now use standard geometric sampling techniques to combine this exact algorithm with our lower bound on depth, resulting in an asymptotically efficient approximation algorithm.

THEOREM 8. Let $d, k$, and $\delta>0$ be constants. Then we can find the a $k$-flat with depth within a $(1-\delta)$ factor of the maximum, for a collection of $n$ points in $\mathbb{R}^{d}$, in time $O(n)$.

PROOF. We first construct an $\epsilon$-approximation $S$ of the points, for the range space consisting of double wedges with one vertical boundary, where $\epsilon=\delta /(2 R(d, k))$. Then if a flat $F$ has depth $D$ with respect to $S, D n /|S|$ is within an additive $\epsilon n$ term of the true depth of $F$ with respect to the original point set. $S$ can be found with $|S|=$ $O\left(\epsilon^{-2} \log \epsilon^{-1}\right)$, in time $O(\epsilon)$, using standard geometric sampling algorithms. We then let $F$ be the deepest flat for $S$.

Suppose the optimal flat $F^{*}$ for the original point set has depth $c n$. Then the depth of $F^{*}$ in $S$, and therefore also the depth of $F$ in $S$, must be at least $(c-\epsilon)|S|$. Therefore, the depth of $F$ in the original point set must be at least $(c-2 \epsilon) n$. Since $c \geq 1 / R(d, k),(c-$ $2 \epsilon) n \geq(1-\delta) c n$.

Although our approximation algorithm takes only linear time, it is likely not practical due to its high constant factors. However, perhaps similar ideas can form the basis of a more practical random sampling based algorithm.

OPEN PROBLEM 6. Improve the time bounds on finding an exact deepest $k$-flat. Is it any easier to find a $k$-flat with depth at least $n / R(d, k)$, that may not necessarily approximate the deepest flat?

\section{ACKNOWLEDGEMENTS}

Work of Eppstein was done in part while visiting Xerox PARC. The authors would like to thank Peter Rousseeuw for helpful comments on a draft of this paper, Ivan Mizera for thoughts on Conjecture 1, and Joel Hass for topological assistance with Open Problem 4.

\section{REFERENCES}

[1] N. Alon and G. Kalai. Bounding the piercing number. Discrete \& Computational Geometry 13(3-4):245-256, 1995.

[2] N. Amenta, M. Bern, D. Eppstein, and S.-H. Teng. Regression depth and center points. Discrete \& Computational Geometry 23(3):305-323, 2000, cs.CG/9809037.

[3] V. L. Dol'nikov. A generalization of the sandwich theorem. Mat. Zametki 52(2):27-37, 1992.
[4] M. Hubert and P. J. Rousseeuw. The catline for deep regression. J. Multivariate Analysis 66:270-296, 1998, http://win-www.uia.ac.be/u/statis/publicat/catline_abstr.html.

[5] M. van Kreveld, J. S. B. Mitchell, P. J. Rousseeuw, M. Sharir, J. Snoeyink, and B. Speckmann. Efficient algorithms for maximum regression depth. Proc. 15th Symp. Computational Geometry, pp. 31-40. ACM, June 1999.

[6] S. Langerman and W. Steiger. An $O(n \log n)$ algorithm for the hyperplane median in $\mathbb{R}^{2}$. Proc. 11th Symp. Discrete Algorithms, pp. 54-59. ACM and SIAM, January 2000.

[7] Z. J. Liu. Robustness of least distances estimate in multivariate linear models. Statistics 23(2):109-119, 1992.

[8] J. Matoušek. Efficient partition trees. Discrete \& Computational Geometry 8(3):315-334, 1992.

[9] I. Mizera. On depth and deep points: a calculus. Inst. Mathematical Statistics Bull. 27(4), 1998, http://www.dcs.fmph.uniba.sk/ mizera/PS/depthps.ps. Full version to appear in Annals of Statistics.

[10] I. Mizera and M. Volauf. Continuity of halfspace depth contours and maximum depth estimators: diagnostics of depth-related methods, http://www.dcs.fmph.uniba.sk/ mizera/PS/mizvolps.ps. Submitted, 2000.

[11] R. Rado. A theorem on general measure. J. London Math. Soc. 21:291-300, 1946.

[12] J. Radon. Mengen konvexer Körper, die einen gemeinsamen Punkt Enthalten. Math. Annalen 83:113-115, 1921.

[13] P. J. Rousseeuw. Least median of squares regression. J. Amer. Statistical Assoc. 79:871-880, 1984.

[14] P. J. Rousseeuw and M. Hubert. Depth in an arrangement of hyperplanes. Discrete \& Computational Geometry 22:167-176, 1999, http://win-www.uia.ac.be/u/statis/publicat/arrang_abstr.html.

[15] P. J. Rousseeuw and M. Hubert. Regression depth. J. Amer. Statistical Assoc. 94(446):388-402, June 1999, http://win-www.uia.ac.be/u/statis/publicat/rdepth_abstr.html.

[16] P. J. Rousseeuw and A. Struyf. Computing location depth and regression depth in higher dimensions. Statistics and Computing 8(3):193-203, August 1998, http: //win-www.uia.ac.be/u/statis/publicat/compdepth_abstr.html.

[17] W. Steiger and R. Wenger. Hyperplane depth and nested simplices. Proc. 10th Canad. Conf. Computational Geometry. McGill Univ., 1998, http://cgm.cs.mcgill.ca/ cccg98/proceedings/cccg98-steiger-hyperplane.ps.gz.

[18] H. Tverberg. A generalization of Radon's theorem. $J$. London Math. Soc. 41:123-128, 1966.

[19] H. Tverberg. A generalization of Radon's theorem II. Bull. Austral. Math. Soc. 24(3):321-325, 1981.

[20] A. C. Yao and F. F. Yao. A general approach to $d$-dimensional geometric queries (extended abstract). Proc. 17th Symp. Theory of Computing, pp. 163-168. ACM, May 1985.

[21] R. T. Živaljević and S. T. Vrećica. An extension of the ham sandwich theorem. Bull. London Math. Soc. 22(2):183-186, 1990. 